\begin{tabular}{cc}
\hline & International Journal of Engineering \& Technology, $7(2.17)(2018) 63-69$ \\
SPC & International Journal of Engineering \& Technology \\
& Website: ww.sciencepubco.com/index.php/IJET \\
Research Paper
\end{tabular}

\title{
Analysis of MRI Data of Brain for CAD System
}

\author{
Girish D Bonde ${ }^{1 *}$, Dr. Manish Jain² \\ ${ }^{1}$ PhD Scholar, SRK University, Bhopal \\ ${ }^{2} \mathrm{HOD}$, EC, RKDF IST, Bhopal \\ *Email: girishbonde55@gmail.com
}

\begin{abstract}
Magnetic resonance imaging (MRI) technologies are currently one of the most effective tools in the diagnosis of a wide variety of socially significant pathologies including cancer, arteriosclerosis, episodes. Ischemic and neurodegenerative diseases [1, 2, 3, 4].This paper gives detailed idea of pre-processing, and segmentation(FCM, soft and hard) of MRI brain tumor images. This paper also insights the machine learning(SOM, NN and SVM) approach for automatic classification(PTPSA, fBM) of brain tissues. Different performance evaluation parameter and similarity metrics are discuss to define the efficiency of computer-aided diagnostic (CAD) system.
\end{abstract}

Keywords:MRI; PTPSA; fBM; CAD, SOM; NN.

\section{Introduction}

There is a great variety of MRI approaches that can be applied in each case, such as images weighted in $\mathrm{T} 1, \mathrm{~T} 2, \mathrm{~T} 2 *$, Magnetization Transfer, Diffusion or Perfusion [5]. However, in many cases the early detection of pathologies by simple visual inspection of the images obtained can be a difficult task. The use of automatic MRI analysis tools is usually very helpful in such cases, allowing a timely diagnosis to effectively treat the pathology [6]. As a consequence, the automatic processing of biomedical images and signals has become one of the most important fields in artificial vision. The fundamental objective of the projects in this field of research is to improve the obtaining of medical information, in such a way that this allows the specialists to reach more accurate, reliable diagnoses and in less time. At present, the technique of Statistical Parametric Mapping (SPM) is the most widespread of automatic analysis of brain images [7]. This technique is general, allowing to analyze different types of experiments. The SPM aims to generate parametric maps that are able to reveal specific brain activation events obtained by functional imaging methods. It is based on mathematical formalisms and biological knowledge that have been developed over time and that are necessary to master to achieve a correct analysis of the images and a consequent interpretation of the results obtained. However, this technique depends to a large extent on the extensive pre-processing that is carried out on the images (noise filtering, alignment, normalization, etc.), in many cases requiring the intervention of a specialist. Similarly, the results obtained with it depend on said pre-processing and prior knowledge of the theory on which it is based. In addition, this technique does not directly solve the problem of classification of images into pathological categories, although it does identify areas where activation may be a case of interest or caused by some pathology.

Fundamental methods of investigation: The documentary analysis that allows to deepen in the particularities, scope, and actuality of the use of techniques of artificial intelligence and pattern recogni- tion for the automatic analysis of medical images. The analysis and synthesis as a method of decomposition of the phenomenon under study that allows knowing the particularities of the parts and the integration of the essential moments of each one of them in an organic whole for the conception of the theoretical framework. After implementing the new method, statistical methods will be used to evaluate the results of its use.

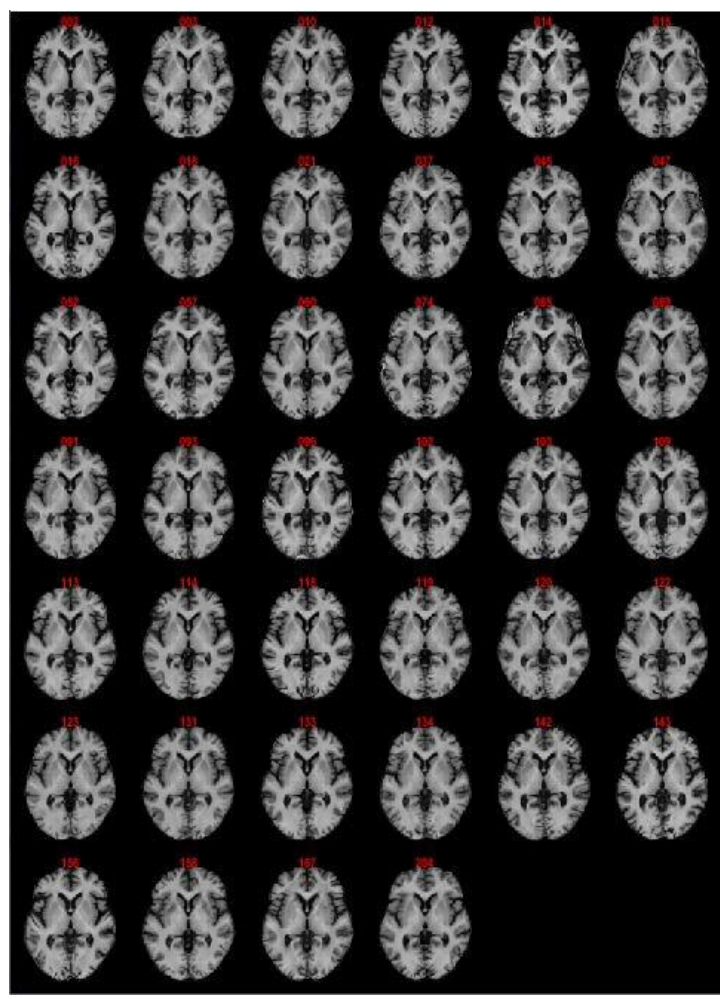

Fig. 1: Display one slice for all images 
This paper is divided into three sections. In the first section, as a summary of the bibliographic review, the generalities of the techniques of pre-processing of biomedical images. In addition, this first section also explains the most important techniques of automatic MR image analysis of the brain, according to its success rate, advantages and disadvantages. In the second section the fundamental characteristics and basic concepts on which the SPM software is based are presented and the results obtained using this software are presented and analyzed for the study of the three brain MR image databases with which counted for the development of this work. Finally, the third section is dedicated to the new method of automatic classification between brain MR images in fasting and feeding states, making reference to the materials and methods used and to the discussion of the effectiveness achieved in its application to each of the three bases of available data.

The automatic processing of images is a problem of great complexity due to the great dimensionality of the data and the different factors that reduce the quality of the images (noise, artifacts, interferences, distortions) and that make it difficult to extract valid information from them. The techniques used in the automatic processing of images can be divided into two groups: techniques that are applied for the pre-processing of the images, and techniques that are used for the search, recognition and extraction of information of interest to them. In the scientific literature on automatic processing of medical images, and more precisely MR images. Therefore, it is necessary to carry out a systematic and critical study to establish the advantages and disadvantages of each of these techniques and to be able to select the most appropriate in each case. This chapter explains a selection of these techniques, chosen for their current relevance and good results.

\section{Biomedical Imaging Pre-Processing Techniques}

Medical images are obtained from the physical interaction of different types of energy with biological tissue [8], in this procedure several factors influence (electromagnetic radiation, acquisition time, variation of electrical energy, the subject examined, etc. [9] that are difficult to control and that can cause the images to contain random noise, artifacts [10], differences in contrasts, deformations, etc., making it difficult to interpret them automatically. These factors are treated in the first phase of image processing, called pre-processing. In this section we will discuss the generalities of a set of techniques that are used in the pre-processing phase of biomedical images grouped into four categories: alignment techniques, filtering techniques, contrast improvement techniques and segmentation techniques [11].

\subsection{Alignment Techniques}

Among the group of techniques that are applied to pre-process images, one of the fundamental medical imaging is alignment or registration $[5,8]$. The alignment or registration of images arises in several fields of research as a solution to the comparison of series of images. Among these fields, medicine stands out, where diagnostic imaging is a fundamental tool in many specialties. In most medical studies it is necessary to compare several images, for example because they are of different modalities (images of MR and CT, among others) or because we want to analyse the evolution over time of a disease in the same patient [13]. The alignment of images consists of determining the necessary transformation to match the coordinates of an image with the coordinates of another or other images that may be representing the same object or similar objects. The entry to a registration algorithm is the set of images to be aligned, the task of the algorithm being to find the transformation that maps the points in one image to the corresponding points in the other. The alignment is good insofar as it adequately maps all the corresponding points. In the case of biomedical images, alignment represents a crucial factor in their analysis and diagnostic interpretation. This process is complicated since, among other circumstances, the images may have been taken at different scales or orientation, and / or under different acquisition conditions, and / or in different subjects whose anatomy may not be identical. Many investigations have focused on medical image alignment techniques $[14,15,16]$, always with the purpose of finding new methods or extending existing ones in order to obtain more accuracy and robustness in the alignment. In general, most of the alignment methods can be analysed as different combinations of: the information that will be used to perform the alignment (methods based on equivalent characteristics, based on segmented structures, based on volumetric methods), the search space or transformation (rigid and non-rigid transformations), the measure of similarity and the search strategy. The study of each of these components for the problem to be solved determines the design of the alignment algorithm to be used. Various criteria have been developed for the similarity metric. Historically, metrics have been studied such as the mean of intensity differences, correlation, metrics based on information theory, the coefficient of variation of intensity ratios, and others [17]. Among them, the criterion of mutual information seems to be the best and has been one of the criteria of similarity most used in recent years, since it allows resolving the alignment of images of different modalities, and is also far superior to other similarity criteria, taking into account counts the quality of the final alignment. There are different variants of this similarity metric [18, 19and 20], such as, for example, the normalized entropy that offers greater efficacy against some types of multimodality alignment.

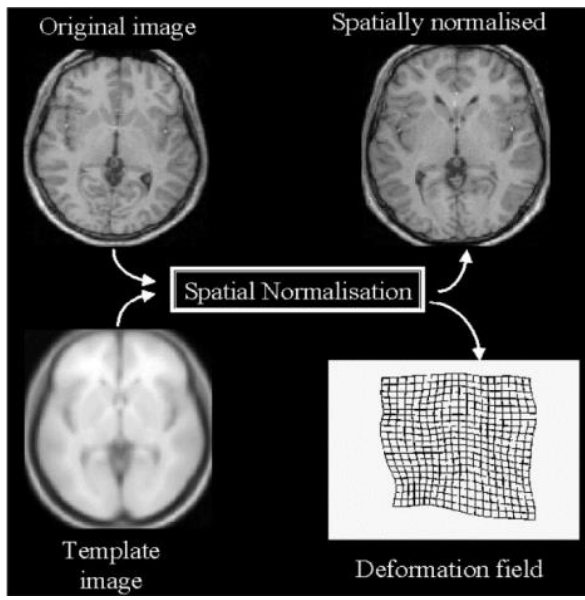

Fig. 2: Normalization of the image

The metric based on mutual information was calculated in the first works by means of the statistical distribution of levels of grey, without taking into account the spatial information. This is equivalent to assuming that the intensity level of each pixel is independent of its neighbours, which is false in images with structures, such as medical images. Recently, some scientific articles [21] propose to extend this metric to take into account the spatial structure of the images. This makes the quality of the alignment is better, however it is necessary to continue validating this approach with some of the field benchmark databases. The non-rigid alignment is another of the topics that has motivated the scientific activity in the line of investigation of the techniques of alignment of 
medical images. If the problem is addressed directly, good alignment is not possible. Among the methods of non-rigid alignment are those dedicated to the alignment between an anatomical atlas and a resonance image. This type of alignment is relevant since it would automatically detect the different structures of the patient, according to the atlas. However, this type of problems is most often affected by the result of previous segmentation. There are works $[22,23]$ that use probabilistic tissue maps (white matter grey matter, cerebrospinal fluid) and adaptive maps that seem to improve alignment. Although there are many alignment algorithms developed, it is still an open research area, especially in cases of non-rigid alignment and inter-modality, because the alignment methods that give good results in one type of images generally fail in another type. Several image processing techniques are presented in [49]-[53].

\subsection{Noise Reduction Techniques}

Noise is part of the information that is contained in medical images and appears in the process of acquiring and digitizing the images [24]. There are several filtering techniques that reduce the noise in the images in order to facilitate the effective analysis of the information of interest in them. Next, some of the most used filtering methods will be discussed. Median filtering Median filtering operates on the image by changing the intensity value of each pixel analyzed, taking into account the intensity values of the pixels in the established neighborhood. The main function of the median filtering is to make the points of very different intensities become very similar to their neighbors, eliminating points of very high and very low intensity [25].

Wiener filtering Assuming that the image s $(x, y)$ is contaminated with white Gaussian noise $\mathrm{w}(\mathrm{x}, \mathrm{y})$, we want to estimate the signal $\mathrm{s}(\mathrm{x}, \mathrm{y})$, from the image contaminated by noise a $(\mathrm{x}, \mathrm{y})$, by observation of the noise by adaptive linear filtering [26].

\subsection{Contrasts Improvement Techniques}

Generally, most medical images have very little contrast which can make it difficult to later detect the effects of interest that may be contained in them. To improve the contrast of images without increasing the noise of the same have developed a large number of techniques that can be classified into two types: direct techniques, which work directly on the image, and indirect techniques, which use indirect means such as histogram [27]. Direct techniques to improve contrast can be based on the application of filters or masks or on multi-resolution methods to capture the morphology of the image. Mask-based methods subtract from the input image a filtered version using a low-pass filter thereof, or add a filtered version using a high-pass filter [28]. It is not advisable to apply linear filters because they greatly increase the noise in the image as opposed to the application of non-linear filters that obtain better results. Among the nonlinear filters, we highlight the modified Laplacian filter based on the local median [29], the use of robust quadratic filters [30] and the use of cubic filters [31]. On the other hand, direct contrast enhancement techniques based on the use of multi-resolution methods and morphological operations use Laplacian image pyramids [32], sub-band encoding procedures [33] and transformed wavelets [34]. In turn, morphological operators are used that are applied directly on the image in their representation at different scales. Among the indirect techniques for contrast enhancement in images, the most popular and simple is the histogram equalization [38]. The histogram equalization modifies an image such that its histogram has a desired shape, using a non- linear monotonic rescaling, which reallocates the intensity values of the pixels of the input image so that the output image contains a uniform distribution of intensities. Due to the redistribution of the probability of occurrence of gray levels in a uniform manner, the perception of the details of the image improves. However, this technique is susceptible to increasing the signal-to-noise ratio, which is why several extensions have been developed, such as the equalization of two histograms, preserving the mean and the dual histogram equalization for equal areas that slightly improve the results [35].

Since the contrast in an image changes significantly throughout the image, adaptive techniques have also been developed that equalize the histogram only of one window around each pixel [36] The quality of these techniques depends strongly redistribute the probabilities of gray levels that are used in each local histogram. The application of a technique or another of improvement of contrasts depends on the concrete problem that is had, that is to say, of the modality of medical image, the type of study (inter-subject or intra-subject), the anatomical area and the pathology that is investigated, etc.

\subsection{Segmentation Techniques}

The segmentation of images is defined as the partition of an image into non-overlapping constituent regions, which are homogeneous with respect to some feature such as intensity or texture.

- Soft segmentation and partial volume effects segmentations that allow regions or classes to overlap are called soft segmentations $[37,38]$. Soft segmentations are important in the treatment of medical images due to the effects of partial volume, where multiple tissues contribute to a single pixel or voxel, resulting in a mixture of intensities at the borders.

- Hard Segmentation:It forces to make a decision as to whether the pixel is inside or outside the object. Soft segmentations, on the other hand, retain more information from the original image allowing ambiguity in the location of the boundaries of the objects.

- Discrete or Continuous Segmentation: Almost all medical images where segmentation is used are represented as discrete samples in a uniform mesh [39].

Segmentation methods typically operate on the same discrete mesh as the image. The pixels are in a discrete spatial domain. If a method can operate values in the continuous spatial domain, then it can operate on the values between two contiguous pixels. In this case, it is said that the method has sub-pixel accuracy [40]. Some methods such as deformable models are able to operate in the continuous spatial domain, providing sub-pixel precision in the delineation of structures. The sub-pixel precision is desirable when the resolution of an image is of the same order of magnitude as the structure of interest.

A great variety of segmentation techniques have been developed and the choice of one or the other depends greatly on the specific application, type of image, and other factors. For example, the requirements of a brain tissue segmentation procedure are different from those of a lung segmentation procedure. There is no segmentation method that achieves acceptable results for all types of medical imaging and specialized methods for specific applications can obtain better results taking into account a priori knowledge. 


\section{Techniques of Automatic Analysis of Mag- netic Resonance Images of the Brain}

In general, most techniques of automatic analysis of medical images of the brain can be divided into two groups: those that work with images of the whole brain and those that segment possible areas of interest. [41] The SPM software is the main representative of the first group and until now the most general and widespread technique in this field. The next chapter of this report will be devoted to explaining the theory behind the SPM and will comment on the results obtained by applying this technique in the images of each of the three databases that were used to carry out this work.

Regarding the techniques that segment possible areas of interest, many have been developed and support their automatic analysis in machine learning methods. In this work a selection of recent publications was made in order to have a global vision of the applicability and success rate of these techniques, paying special attention to methods of detection and classification of brain activations and pathologies.

\section{Classification of Tissue Types or Region of Interest in MRI}

Accurate classification of tissue types or regions of interest in MR images has become a fundamental requirement in diagnosis, treatment planning, and cognitive neuroscience. Several authors have shown that finite mixture models give excellent results in the automatic segmentation of MR images of the normal human brain; in particular, finite methods based on Gaussian mixtures have gained wide acceptance [42].

\section{Detection and Classification of Brain Tu- mors in MRI}

Intensity is an important feature for the detection of tumors automatically, however, it is not enough with this single characteristic to achieve satisfactory segmentations and classifications. A research was published on the efficacy of the fusion of two texture characteristics based on fractals together with the intensity characteristic in multimodal MR images for the segmentation of pediatric brain tumors and their classification [43]. In this research the characteristics of fractal texture and fractal-wavelet were used. The fractal feature describes the geometric complexity of objects in nature. The fractal dimension is a real number that represents the fractal property of the objects. In [44], the fractal feature is extracted with the Piecewise-Triangular-Prism-Surface-Area (PTPSA) algorithm [45], while the fractal-wavelet is obtained using a framework based on the Fractional Brownian Motion (fBm) [46] that combines both wavelet and fractal analysis. The algorithm of segmentation and classification of pediatric brain tumors, which combines the characteristic of intensity with fractal characteristics, has the following general steps:

1. Normalize the intensity values of the images in the three modes.

2. Extract the three types of characteristics: intensity, fractal and fractal-wavelet of the normalized images.

3. The characteristics are fused and the groups of tumor segmentations are obtained using a neural network SelfOrganizing Maps (SOM) [47].
4. After segmentation, the groups are manually labeled by a specialist as tumor or non-tumor.

5. The labelled segments are divided into training and test sets to construct a feed forward multilayer classifier for each of the correctly segmented patient data sets.

The algorithm was tested with a total of 204 MR images in 2D and three modalities: T1 with enhanced contrast, T2 and FluidAttenuated Inversion-Recovery; obtained from nine sick pediatric patients. Multimodal MR images were segmented and in a single modality. The success of the tumor segmentation was $100 \%$ for the first case, not so in the second case. The experimental results suggest that the fusion of fractal, fractal-wavelet and intensity characteristics in multimodal MR images offers better results of tumor segmentation compared to those obtained using only fractal and intensity characteristics in a single modality of images of MR. They also used a multi-layer feed forward neural network with automatic Bayesian regularization to classify the tumor regions of the non-tumor regions. They obtained the ROC curves to evaluate the performance of the classification, and they indicated that for a threshold value of 0.7 , the values of the rate of true positives (TPF) ranged from $75 \%$ to $100 \%$ for different patients, with an average value of $90 \%$. Notwithstanding the relevant results obtained with this algorithm, it is observed that such results are to some extent dependent on the patient, that is, when the images are from the same patient, the segmentation and classification of the tumors is more effective than with images of different patients. Another difficulty is the need for user intervention to manually label the segmentations, which makes the process not fully automatic. On the other hand, the authors of this algorithm stated that they made a detailed study of the segmentations of the resulting tumors and realized that the vectors of characteristics used may not be sufficient to discriminate several types of brain tissues such as white matter, gray matter, cerebrospinal fluid and the skull of a solid tumor and edema. Therefore, they foresee the need to include additional features to discriminate and classify tumors from multiple tissues.

\section{Automatic Systems for Diagnosing of Brain Diseases in MR Images}

The development of computer-aided diagnostic (CAD) systems using MR images has been more difficult than with other medical images since MR image quality includes specific artifacts and noise in the image that can vary according to the sequences of images and patients. However, in recent years several types of $\mathrm{CAD}$ methods have been developed in neuroradiology using MR images with favorable results [48], for example in the detection of brain diseases such as asymptomatic aneurysms, Alzheimer's, vascular dementia and multiple sclerosis. CAD systems consist in the extraction of image characteristics based on image processing techniques and machine learning techniques such as linear discriminant analysis, artificial neural networks, and support vector machines. These systems suggest a second opinion for neuroradiologists that serves to corroborate their diagnoses or help them reach them in case of doubts.

In general, there are two types of CAD systems, depending on the number of classes in which they classify the data set. Binary classification CAD systems (two classes) can be considered, which classify all candidates into abnormal and normal candidates, for example in cases of diseases such as intracranial aneurysms or 
hyper intensities of white matter in MR images. The other type of CAD system is the one that makes a multi-class classification of unknown cases in various types of anomalies. For example, the automatic classification of brain tumors in high-grade gliomas, metastatic brain tumors, low-grade glioma, malignant lymphoma, etc.

The CAD systems are formed by a pre-processing phase (normalization, noise elimination, alignment, segmentation), extraction of characteristics (depending on the type of application and the objective for which the CAD system is designed) and classification. Although the experimental results they show are favorable in a general way, they can be affected by the pre-processing phase, the selection of characteristics and the segmentation of possible areas of interest. In addition, the comparisons of the methods that are made in this article are not using the same database of MR images, which restricts the level of the conclusions that can be reached.

\section{Performance Evaluation Metrics}

This section describes the evaluation metrics used to assess the algorithms designed, analyzing the segmented region, the contour of the region segmented, the computational cost and the volume. To assess whether the algorithms proposed correctly classify the voxels belonging to the tumor'sretroperitoneal images in the CAT images, the following must be calculated four variables:

1. True positive (TP): the voxel belongs to the tumor according to the truth of reference and the designed algorithm thus determines it.

2. False positive (FP): the voxel does not belong to the tumor according to the truth of reference but the algorithm determines that it does belong.

3. True negative (TN): the voxel does not belong to the tumor according to the truth of reference and the designed algorithm so determines it.

4. False negative (FN): the voxel belongs to the tumor according to the reference truth, but the algorithm considers that it is not a tumor.

A good diagnosis is the one that detects retroperitoneal tumors in the voxels in where tumors exist, that is, the one that maximizes both true positives and true negatives.

\section{Simulation Results}

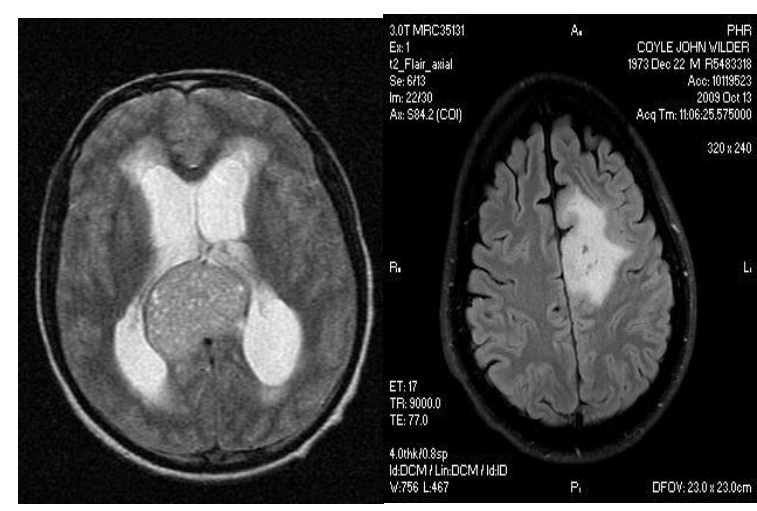

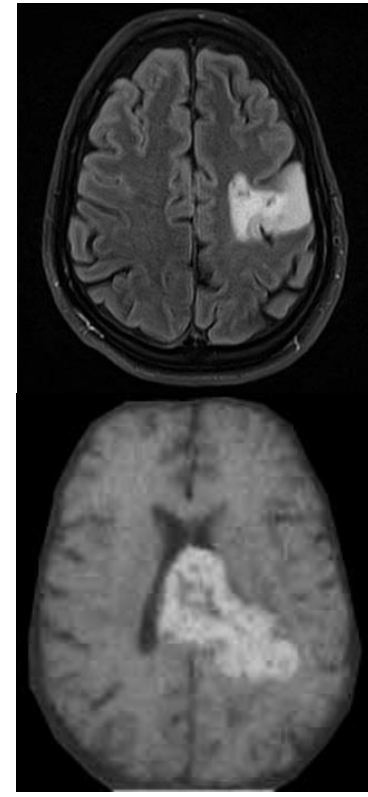

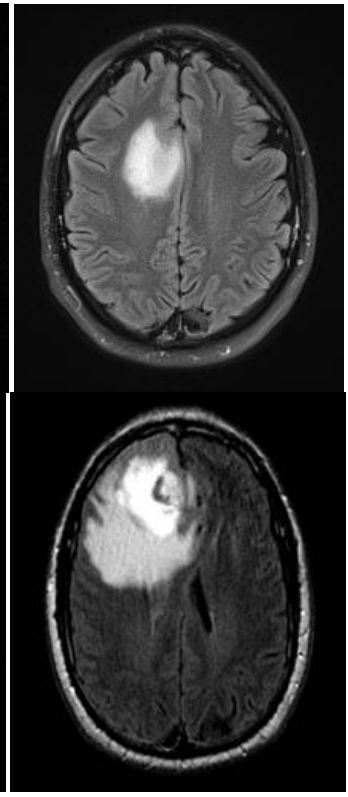

Fig. 3: Input images

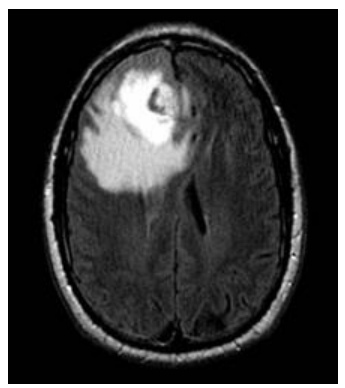

Figure 4(a)

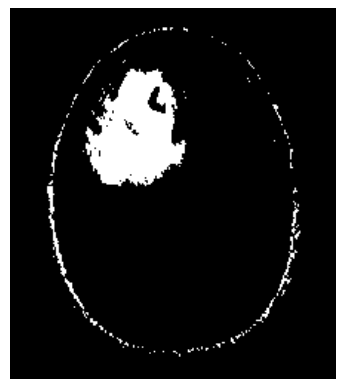

Figure 4(c)

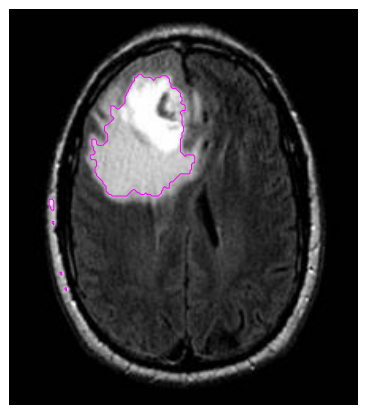

Figure 4(e)

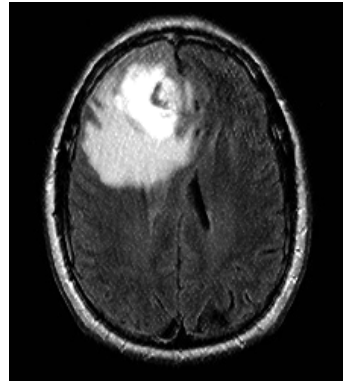

Figure 4(b)

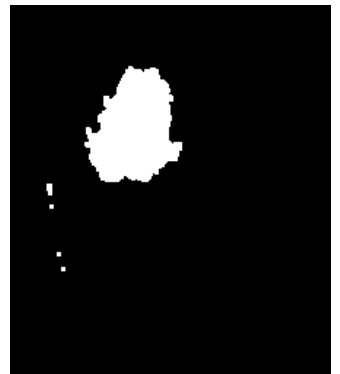

Figure 4(d)

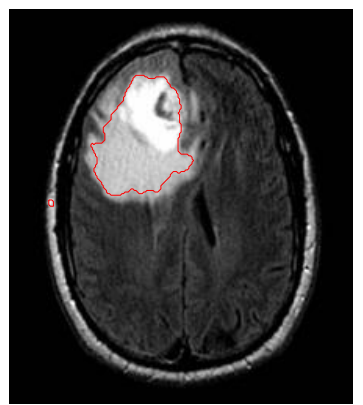

Figure 4(f)
Fig. 4(a): High pass filtered image, 4(b): Enhanced image, 4(c): Binary Image, 4(d): Morphed image with detected tumour region 4(e): Original image with initial tumour region, $\mathbf{4 ( f )}$ : Original image with tumour region 


\section{Conclusion}

The techniques of pre-processing of medical images and the techniques of automatic analysis of medical images, paying special attention to the most recent and significant that have been applied to brain images obtained by MR. Among the techniques of preprocessing of the images, those related to alignment, noise reduction, improvement of contrasts and segmentation of areas of interest were discussed. All these procedures are based on extensive and complex mathematical developments that include different parameters and variables, which almost always end up being approximated in order to apply the technique to a real problem.

There are a variety of methods that can be used at different times in the pre-processing phase of medical images, all with different requirements and objectives. In addition, the performance of each of these methods is generally tested individually, so that each article that is published uses different test environments and evaluation metrics. This paper also elaborates the, automatic analysis techniques of brain MR images, chosen for their contributions in this branch of image processing, were described. These methods are based on machine learning algorithms and generally make a prior segmentation of certain areas of the brain where it is estimated that an anomaly may be occurring. Segmentation has its advantages because it allows to concentrate on the analysis of a specific area of the images and less information is processed. However, it is a cumbersome process due to its inaccuracy and can result in errors in subsequent steps of image analysis. In MR images of the brain, special care must be taken with segmentation procedures for areas of interest, as these images are difficult to analyse due to their high degree of noise and devices dependent on the equipment and the patient. Despite this, it has been confirmed in the review that there are segmentation methods that yield good results, This is important because, in general, the pathologies are related to anomalies in these areas, for example, in cases of diseases such as intracranial aneurysms, it is identified by hyper intensities of white matter in MR images.

Based on these theoretical foundations, it was decided to implement a method of automatic analysis of MR images of the brain, using machine learning algorithms that do not require extensive pre-processing of the images, in order to detect differences that allow automatic classification.

\section{Acknowledgement}

The authors would first like to thank the brainweb (http://www.bic.mni.mcgill.co/brainweb), for providing brain MR images. Also, they would like to thank the reviewers and editors for their very helpful suggestions and insightful comments which have resulted in an improved paper.

\section{References}

[1] Baker, A.D., 2014. Abnormal magnetic-resonance scans of the lumbar spine in asymptomatic subjects. A prospective investigation. In Classic Papers in Orthopaedics (pp. 245-247). Springer, London.

[2] Muller, B.G., Fütterer, J.J., Gupta, R.T., Katz, A., Kirkham, A., Kurhanewicz, J., Moul, J.W., Pinto, P.A., Rastinehad, A.R., Robertson, C. and Rosette, J., 2014. The role of magnetic resonance imaging (MRI) in focal therapy for prostate cancer: recommendations from a consensus panel. BJU international, 113(2), pp.218-227.

[3] Fatahi, M. and Speck, O., 2015. Magnetic resonance imaging (MRI): A review of genetic damage investigations. Mutation Research/Reviews in Mutation Research, 764, pp.51-63.
[4] Rosenkrantz, A.B., Verma, S., Choyke, P., Eberhardt, S.C., Eggener, S.E., Gaitonde, K., Haider, M.A., Margolis, D.J., Marks, L.S., Pinto, P. and Sonn, G.A., 2016. Prostate magnetic resonance imaging and magnetic resonance imaging targeted biopsy in patients with a prior negative biopsy: a consensus statement by AUA and SAR. The Journal of urology, 196(6), pp.1613-1618.

[5] Burth, S., Kickingereder, P., Eidel, O., Tichy, D., Bonekamp, D., Weberling, L., Wick, A., Löw, S., Hertenstein, A., Nowosielski, M. and Schlemmer, H.P., 2016. Clinical parameters outweigh diffusion-and perfusion-derived MRI parameters in predicting survival in newly diagnosed glioblastoma. Neurooncology, 18(12), pp.1673-1679.

[6] Prager, A.J., Martinez, N., Beal, K., Omuro, A., Zhang, Z. and Young, R.J., 2015. Diffusion and perfusion MRI to differentiate treatment-related changes including pseudoprogression from recurrent tumors in high-grade gliomas with histopathologic evidence. American Journal of Neuroradiology, 36(5), pp.877-885.

[7] Kurth, F., Gaser, C. and Luders, E., 2015. A 12-step user guide for analyzing voxel-wise gray matter asymmetries in statistical parametric mapping (SPM). Nature protocols, 10(2), p.293.

[8] Suetens, P., 2017. Fundamentals of medical imaging. Cambridge university press.

[9] Bushong, S.C. and Clarke, G., 2014. Magnetic resonance imaging: physical and biological principles. Elsevier Health Sciences.

[10] Fowler, K.J., Maxwell, J., Saad, N.E., Yano, M., Raptis, C., Menias, C. and Narra, V., 2014. Magnetic resonance imaging of iatrogeny: understanding imaging artifacts related to medical devices. Abdominal imaging, 39(2), pp.411-423.

[11] DU, R., Chiu, W.H.K., Lee, E.Y.P., Pang, H.M.H., Lam, E.Y.M. and Vardhanabhuti, V., 2017. Inter-session reproducibility and consistency of radiomic features after preprocessing as methods for quality control in MRI quantitative radiomics. In 7th Joint Scientific Meeting of The Royal College of Radiologists \& Hong Kong College of Radiologists and 25th Annual Scientific Meeting of Hong Kong College of Radiologists.

[12] Silva, A., Pinto, E. and Sampaio, R., 2016. Rotational alignment in patient-specific instrumentation in TKA: MRI or CT?. Knee Surgery, Sports Traumatology, Arthroscopy, 24(11), pp.36483652 .

[13] Silva, A., Pinto, E. and Sampaio, R., 2016. Rotational alignment in patient-specific instrumentation in TKA: MRI or CT?. Knee Surgery, Sports Traumatology, Arthroscopy, 24(11), pp.36483652.

[14] McKenna, B.S., Theilmann, R.J., Sutherland, A.N. and Eyler, L.T., 2015. Fusing functional MRI and diffusion tensor imaging measures of brain function and structure to predict working memory and processing speed performance among inter-episode bipolar patients. Journal of the International Neuropsychological Society, 21(5), pp.330-341.

[15] Crema, M.D., Cortinas, L.G., Lima, G.B., Abdalla, R.J., Ingham, S.J.M. and Skaf, A.Y., 2018. Magnetic resonance imaging-based morphological and alignment assessment of the patellofemoral joint and its relationship to proximal patellar tendinopathy. Skeletal radiology, 47(3), pp.341-349.

[16] Cárdenas-Peña, D., Collazos-Huertas, D. and CastellanosDominguez, G., 2016. Centered kernel alignment enhancing neural network pretraining for MRI-based dementia diagnosis. Computational and Mathematical Methods in Medicine, 2016.

[17] França, L.K.L., Bitencourt, A.G.V., de Toledo Osório, C.A.B., Graziano, L., Guatelli, C.S., Souza, J.A. and Marques, E.F., 2018 Tumor size assessment of invasive breast cancers: which pathological features affect MRI-pathology agreement?. Applied Cancer Research, 38(1), p.2.

[18] Weller-Fahy, D.J., Borghetti, B.J. and Sodemann, A.A., 2015. A survey of distance and similarity measures used within network intrusion anomaly detection. IEEE Communications Surveys \& Tutorials, 17(1), pp.70-91.

[19] Larsen, A.B.L., Sønderby, S.K., Larochelle, H. and Winther, O., 2015. Autoencoding beyond pixels using a learned similarity metric. arXiv preprint arXiv:1512.09300.

[20] Luo, H., Wang, J., Li, M., Luo, J., Peng, X., Wu, F.X. and Pan, Y., 2016. Drug repositioning based on comprehensive similarity measures and Bi-Random walk algorithm. Bioinformatics, 32(17), pp.2664-2671.

[21] Cao, L., Jin, L., Tao, H., Li, G., Zhuang, Z. and Zhang, Y., 2015. Multi-focus image fusion based on spatial frequency in discrete cosine transform domain. IEEE signal processing letters, 22(2), pp. $220-224$. 
[22] Chen, K.T., Izquierdo-Garcia, D., Poynton, C.B., Chonde, D.B. and Catana, C., 2017. On the accuracy and reproducibility of a novel probabilistic atlas-based generation for calculation of head attenuation maps on integrated PET/MR scanners. European journal of nuclear medicine and molecular imaging,44(3), pp.398-407.

[23] Blaiotta, C., Freund, P., Cardoso, M.J. and Ashburner, J., 2018. Generative diffeomorphic modelling of large MRI data sets for probabilistic template construction. NeuroImage, 166, pp.117134.

[24] Manjón, J.V., Coupé, P. and Buades, A., 2015. MRI noise estimation and denoising using non-local PCA. Medical image analysis, 22(1), pp.35-47.

[25] Menon, N. and Ramakrishnan, R., 2015, April. Brain Tumor Segmentation in MRI images using unsupervised Artificial Bee Colony algorithm and FCM clustering. In Communications and Signal Processing (ICCSP), 2015 International Conference on(pp. 0006-0009). IEEE.

[26] Goyal, B., Agrawal, S., Sohi, B.S. and Dogra, A., 2016. Noise Reduction in MR brain image via various transform domain schemes. Research Journal of Pharmacy and Technology, 9(7), pp.919-924

[27] Tan, C.H., Paul Hobbs, B., Wei, W. and Kundra, V., 2015. Dynamic contrast-enhanced MRI for the detection of prostate cancer: meta-analysis. American Journal of Roentgenology, 204(4), pp.W439-W448.

[28] Rosenkrantz, A.B., Geppert, C., Grimm, R., Block, T.K., Glielmi, C., Feng, L., Otazo, R., Ream, J.M., Romolo, M.M., Taneja, S.S. and Sodickson, D.K., 2015. Dynamic contrast-enhanced MRI of the prostate with high spatiotemporal resolution using compressed sensing, parallel imaging, and continuous golden-angle radial sampling: Preliminary experience. Journal of Magnetic Resonance Imaging, 41(5), pp.1365-1373.

[29] Pitchammal, M., Nisha, S.S. and Sathik, M.M., 2016. Noise Reduction in MRI Neck Image Using Adaptive Fuzzy Filter in Contourlet Transform. International Journal of Engineering Science, 2478.

[30] Kagoiya, K. and Mwangi, E., 2017. A hybrid and adaptive nonlocal means wavelet based MRI denoising method with bilateral filter enhancement. International Journal of Computer Applications, 166(10).

[31] Joseph, J., Sivaraman, J., Periyasamy, R. and Simi, V.R., 2016 Noise based computation of decay control parameter in nonlocal means filter for MRI restoration. Journal of Medical Imaging and Health Informatics, 6(4), pp.1027-1037.

[32] Ali, H., Elmogy, M., El-Daydamony, E. and Atwan, A., 2015. Multi-resolution MRI brain image segmentation based on morphological pyramid and fuzzy c-mean clustering. Arabian Journal for Science and Engineering, 40(11), pp.3173-3185.

[33] Deshmukh, P. and Malge, P.S., 2016. Classification of Brain MRI using Wavelet Decomposition and SVM. Entropy, 1(2), p.5.

[34] Kanade, P.B. and Gumaste, P.P., 2015. Brain tumor detection using MRI images. Brain, 3(2).

[35] Teh, V., Sim, K.S. and Wong, E.K., 2016, January. ExtremeLevel Eliminating Brightness Preserving Bi-Histogram Equalization Technique for Brain Ischemic Detection. In Proceedings of the International Conference on Image Processing, Computer Vision, and Pattern Recognition (IPCV)(p. 69). The Steering Committee of The World Congress in Computer Science, Computer Engineering and Applied Computing (WorldComp).

[36] Gupta, S., Gupta, R. and Singla, C., 2017. Analysis of image enhancement techniques for astrocytoma MRI images. International Journal of Information Technology,9(3), pp.311-319.

[37] Despotović, I., Goossens, B. and Philips, W., 2015. MRI segmentation of the human brain: challenges, methods, and applications. Computational and mathematical methods in medicine, 2015.

[38] Pereira, S., Pinto, A., Alves, V. and Silva, C.A., 2016. Brain tumor segmentation using convolutional neural networks in MR images. IEEE transactions on medical imaging, 35(5), pp.12401251 .

[39] Menon, N. and Ramakrishnan, R., 2015, April. Brain Tumor Segmentation in MRI images using unsupervised Artificial Bee Colony algorithm and FCM clustering. In Communications and Signal Processing (ICCSP), 2015 International Conference on(pp. 0006-0009). IEEE.

[40] Adhikari, S.K., Sing, J.K., Basu, D.K. and Nasipuri, M., 2015 Conditional spatial fuzzy C-means clustering algorithm for seg- mentation of MRI images. Applied Soft Computing, 34, pp.758769

[41] Salimi-Khorshidi, G., Douaud, G., Beckmann, C.F., Glasser, M.F., Griffanti, L. and Smith, S.M., 2014. Automatic denoising of functional MRI data: combining independent component analysis and hierarchical fusion of classifiers. Neuroimage, 90, pp.449-468.

[42] Jiang, Y., Zhu, C., Peng, W., Degnan, A.J., Chen, L., Wang, X., Liu, Q., Wang, Y., Xiang, Z., Teng, Z. and Saloner, D., 2016. Ex-vivo imaging and plaque type classification of intracranial atherosclerotic plaque using high resolution MRI. Atherosclerosis, 249, pp.10-16.

[43] Chavan, N.V., Jadhav, B.D. and Patil, P.M., 2015. Detection and classification of brain tumors. International Journal of Computer Applications, 112(8).

[44] Antonio Di Ieva, M.D., Pierre-Jean Le Reste, M.D., Carsin-Nicol, B., Ferre, M.J.C. and Cusimano, M.D., 2016. Diagnostic Value of Fractal Analysis for the Differentiation of Brain Tumors Using 3-Tesla Magnetic Resonance Susceptibility-Weighted Imaging.

[45] Saravanan, G. and Krishnamoorthy, K., 2014. An Enhanced Implementation PTPSA Algorithm for Fractal Feature Extraction and Abnormal Tissue Segmentation.

[46] Chinnasamy, G. and Vanitha, S., 2015. Fractional brownian motion and fractal analysis of brain mri images: a review. IJAR, 1(3), pp.21-24

[47] Vaishnavee, K.B. and Amshakala, K., 2015, March. An automated MRI brain image segmentation and tumor detection using SOM-clustering and Proximal Support Vector Machine classifier. In Engineering and Technology (ICETECH), 2015 IEEE International Conference on (pp. 1-6). IEEE.

[48] Song, S.E., Seo, B.K., Cho, K.R., Woo, O.H., Son, G.S., Kim, C. Cho, S.B. and Kwon, S.S., 2015. Computer-aided detection (CAD) system for breast MRI in assessment of local tumor extent, nodal status, and multifocality of invasive breast cancers: preliminary study. Cancer Imaging, 15(1), p.1

[49] Md Zia Ur Rahman, B.Malakonda Reddy, "Efficient SAR Image Segmentation Techniques using Biasfield Estimation”, Journal of Scientific and Industrial Research, vol. 76, pp. 335-338, 2017

[50] P.V.V. Kishore, A.S.C.S. Sastry, Md. Zia Ur Rahman, "Double Technique for Improving Ultrasound Medical Images”, Journal of Medical Imaging and Health Informatics, vol.6, no.3, pp.667675, 2016.

[51] M. Lakshmi, Md Zia Ur Rahman, "Efficient Speckle Noise Reduction Techniques for Synthetic Aperture Radars in Remote Sensing Applications", International Review of Aerospace Engineering Vol.9, no.10, 2016, pp.114-122.

[52] M. Lakshmi, Md Zia Ur Rahman, "Analysis of Synthetic Aperture Radar Images using Brute Force Thresholding and Gradient Guide Filters", Journal of Theoretical and Applied Information Technology,Vol.93, no.1, 2016, pp.152-163.

[53] B. Mala Konda Reddy, Md. Zia Ur Rahman, "Novel Segmentation Technique for Target Tracking in Synthetic Aperture Radars", International Journal of Control Theory and Applications, Vol.10, no.35, 2017, pp.335-341. 